\title{
Computing the $q$-Numerical Range of Differential Operators
}

\author{
Ahmed Muhammad $\mathbb{D}^{1}$ and Faiza Abdullah Shareef ${ }^{1}$ \\ ${ }^{1}$ Department of Mathematics, College of Science, University of Salahaddin, Erbil, Iraq \\ Correspondence should be addressed to Ahmed Muhammad; ahmed.muhammad@su.edu.krd
}

Received 7 May 2020; Accepted 17 July 2020; Published 28 August 2020

Academic Editor: Ali R. Ashrafi

Copyright (c) 2020 Ahmed Muhammad and Faiza Abdullah Shareef. This is an open access article distributed under the Creative Commons Attribution License, which permits unrestricted use, distribution, and reproduction in any medium, provided the original work is properly cited.

\begin{abstract}
A linear operator on a Hilbert space may be approximated with finite matrices by choosing an orthonormal basis of thez Hilbert space. In this paper, we establish an approximation of the $q$-numerical range of bounded and unbounnded operator matrices by variational methods. Application to Schrödinger operator, Stokes operator, and Hain-Lüst operator is given.
\end{abstract}

\section{Introduction and Definitions}

The simplest concepts which can be used to obtain an enclosure of the spectrum of a linear operator $A$ in a Hilbert space $\mathscr{H}$ are the numerical range.

$$
W(A)=\{\langle A x, x\rangle, \text { for some } x \in \mathscr{D}(A),\|x\|=1=\},
$$

where $\mathscr{D}($.$) denotes the domain. It is not difficult to see that$ the point spectrum $\sigma_{p}(A)$ of $A$ is contained in $W(A)$ and that the approximate point spectrum $\sigma_{\text {app }}(A)$ of $A$ is contained in the closure of $W(A)$; the inclusion $\sigma(A) \subset W \overline{(A)}$ holds if $A$ is closed. Many estimates of eigenvalues of differential operators, for instance, involve calculating estimates of the inner products $\langle A x, x\rangle$, using partial integration. It is always convex [1]. However, the numerical range often gives a poor localization of the spectrum and cannot reveal the existence of spectral gaps. In [2], the numerical range of a (finite) matrix was approximated by projection methods. This concept was generalized to $q$-numerical range in [3], as follows:

$$
\begin{gathered}
W_{q}(A)=\{\langle A x, y\rangle, \text { for some } x, y \in H,\|x\|=1=\|y\|, \\
\langle x, y\rangle=q\},
\end{gathered}
$$

where $0 \leq q \leq 1$. It is easy to see that if $q=1$, then $W_{q}(A)$ coincides with the numerical range of $A$. For a closed operator $A$, the closure of the $q$-numerical range contains the eigenvalues of $A$ scaled by $q$, and $W_{q}(\alpha A+\beta I)=\alpha W_{q}(A)+$ $\beta I$, for every $\alpha, \beta \in \mathbb{C}$. Moreover, It is known that $W_{q}(A)$ is a compact convex subset of $\mathbb{C}[4]$. A review of the properties of the $q$-numerical range of operator matrices may be found in $[3,5,6]$. The main purpose of this work is the approximation of the $q$-numerical range of bounded and unbounded operator matrices, which contains both theoretical results and applications to some self-adjoint and non-self-adjoint operator matrices. The paper is organized as follows. In Section 2, we establish an approximation of the $q$-numerical range of bounded and unbounded operator matrices. In Section 3, we shall apply these results to compute the $q$-numerical range of differential operators and some new analytic bounds.

\section{Convergence Theorems}

In this section, we will use finite matrices to approximate the numerical range of linear operators. However, the idea of approximating linear operators by finite matrices is an obvious one that must happen again and again, suppose that one wishes to compute the $q$-numerical range of $A$ by using the following projection method. Let $\left(\mathscr{L}_{k}\right)_{k=1}^{\infty}$ be a nested family of spaces in $\mathscr{H}$ given by $\mathscr{L}_{k}=\operatorname{span}\left\{\phi_{1}, \phi_{2}, \cdots, \phi_{k}\right\}$, where \{ $\left.\mathscr{L}_{k}: k \in \mathbb{N}\right\}$ is the orthonormal basis of a Hilbert space $\mathscr{H}$ whose element lies in $\mathscr{D}(A)$ and suppose that the corresponding orthogonal projections $P_{k}: \mathscr{H} \longrightarrow \mathscr{L}_{k}$ converge strongly to the identity operator $I$. We identify $A$ with its 
matrix representation with respect to $\left\{\phi_{k}: k \in \mathbb{N}\right\}$ :

$$
A=\left(A_{i j}\right)_{i, j=1}^{\infty}, \quad A_{i j}:=\left\langle A \phi_{j}, \phi_{i}\right\rangle \quad i, j \in N
$$

Then, the compression of $A$ to $\mathscr{L}_{k}$ is denoted by $\mathbb{A}_{k}:=\left.P_{k} A\right|_{\mathscr{L}_{k}}$, where

$$
\mathbb{A}_{k}=\left(\begin{array}{cccc}
\left\langle A \phi_{1}, \phi_{1}\right\rangle & \left\langle A \phi_{1}, \phi_{2}\right\rangle & \cdots & \left\langle A \phi_{1}, \phi_{k}\right\rangle \\
\left\langle A \phi_{2}, \phi_{1}\right\rangle & \left\langle A \phi_{2}, \phi_{2}\right\rangle & \cdots & \left\langle A \phi_{2}, \phi_{k}\right\rangle \\
\vdots & \vdots & & \vdots \\
\left\langle A \phi_{k}, \phi_{1}\right\rangle & \left\langle A \phi_{1}, \phi_{2}\right\rangle & \cdots & \left\langle A \phi_{k}, \phi_{k}\right\rangle
\end{array}\right) .
$$

Theorem 1. Let $A$ be a bounded operator in $\mathscr{H}$ Let $\left\{\mathscr{L}_{k}: k\right.$ $\in \mathbb{N}\}$ be a nested family of spaces in $\mathscr{H}$ given by $\mathscr{L}_{k}=$ span $\left\{\phi_{1}, \phi_{2}, \cdots, \phi_{k}\right\}$ where $\left\{\phi_{k}: k \in \mathbb{N}\right\}$ is orthonormal, and $\mathbb{A}_{k}$ be as in Equation((4)). Then, $W_{q}\left(\mathbb{A}_{k}\right) \subseteq W_{q}(A)$, for $q \in[0,1]$.

Proof. Define an isometry $i: \mathscr{L}_{k} \longrightarrow \mathbb{C}^{k}$ by $i\left(\alpha_{1} \phi_{1}+\alpha_{2} \phi_{2}+\right.$ $\left.\cdots+\alpha_{k} \phi_{k}\right):=\left(\alpha_{1}, \alpha_{2}, \cdots, \alpha_{k}\right)$, and $i\left(\beta_{1} \phi_{1}+\beta_{2} \phi_{2}+\cdots+\beta_{k} \phi_{k}\right):=($ $\left.\beta_{1}, \beta_{2}, \cdots, \beta_{k}\right)$. Suppose that $\lambda \in W_{q}\left(\mathbb{A}_{k}\right)$. Then, for some $\alpha$, $\beta \in \mathbb{C}^{k}$, with $\|\alpha\|=1=\|\beta\|,(\alpha, \beta)=q$ such that $\lambda=\left\langle\mathbb{A}_{k} \alpha, \beta\right\rangle$. Choose $\xi, \eta \in \mathscr{L}_{k}$, such that $i(\xi)=\alpha, i(\eta)=\beta$, and $\|\xi\|=1=\|$ $\eta \|$. Then, a direct computation shows that $\lambda=\langle A \xi$, $\eta\rangle$, where $\xi=\sum_{j=1}^{k} \alpha_{j} \phi_{j}$, and $\eta=\sum_{j=1}^{k} \beta_{j} \phi_{j}$. Thus, $\lambda \in W_{q}(A)$.

The next inclusion, which will be used in the proof of Theorem 3, asserts that $\left\{\left(W_{q}\left(\mathbb{A}_{k}\right)\right): k \geq 2\right\}$ forms an increasing sequence of sets.

Lemma 2. Let $\left\{\mathscr{L}_{k}: k \in \mathbb{N}\right\}$ and $\mathbb{A}_{k}$ be as in Theorem1. Given $q \in(0,1]$, then $W_{q}\left(\mathbb{A}_{k}\right) \subseteq W_{q}\left(\mathbb{A}_{\tau}\right)$, for $r \geq k$.

Proof. This is an immediate consequence of the fact that $\mathbb{C}^{k}$ is a subspace of $\mathbb{C}^{\tau}$. In detail, suppose $\lambda$ is in $W_{q}\left(\mathbb{A}_{k}\right)$; then, there exists $\alpha, \beta \in \mathbb{C}^{k},\|\alpha\|=1=\|\beta\|$, with $\langle\alpha, \beta\rangle=q$ such that $\lambda=\left\langle\mathbb{A}_{k} \alpha, \beta\right\rangle$. Choose $\varsigma, v \in \mathbb{C}^{\tau}$ by setting $\varsigma=$ $\left(\alpha_{1}, \alpha_{2}, \cdots, \alpha_{j}, 0, \cdots, 0\right)^{T}, v=\left(\beta_{1}, \beta_{2}, \cdots, \beta_{j}, 0, \cdots, 0\right)^{T}$. A simple calculation shows that $\langle\varsigma, v\rangle=\langle\alpha, \beta\rangle=q$ and $\left\langle\mathbb{A}_{k} \alpha, \beta\right\rangle=\left\langle\mathbb{A}_{k}\right.$ $\varsigma, v\rangle$ and so $\lambda$ is in $W_{q}\left(\mathbb{A}_{\tau}\right)$.

Theorem 3. Let $A$ and $\mathbb{A}_{k}$ be as in Theorem1. Let $P_{k}: \mathscr{H}$ $\rightarrow \mathscr{L}_{k}$ be orthogonal projection. If $P_{k}$ converge strongly to the identity operator $I$, then $W_{q}^{-}(A)=\bigcup_{j=1}^{\infty} \bar{W}_{q}\left(\mathbb{A}_{j}\right)$.

Proof. In view of Theorem 1 , it is sufficient to prove $W_{q}(A)$ $\subseteq \bigcup_{j=1}^{\infty} \bar{W}_{q}\left(\mathbb{A}_{j}\right)$. Suppose $\lambda \in W_{q}(A)$. Choose $x, y \in \mathscr{H}$ such that $\|x\|=\|y\|=1$ and $\langle x, y\rangle=q$, such that $\lambda=\langle A x, y\rangle$. We know $P_{k} x \longrightarrow x$ and $P_{k} y \longrightarrow y$ as $k \longrightarrow \infty$ and if $x_{k}:=i\left(P_{k} x\right)$, $y_{k}:=i\left(P_{k} y\right)$ thus $\left\langle A x_{k}, y_{k}\right\rangle \longrightarrow\langle A x, y\rangle$ as $k \longrightarrow \infty$ and $\left\langle x_{k}\right.$, $\left.y_{k}\right\rangle \longrightarrow\langle x, y\rangle=q$ as $k \longrightarrow \infty$.
Fix $k>0$. Let $i: \operatorname{span}\left\{\phi_{1}, \phi_{2}, \cdots, \phi_{s_{k}}\right\} \longrightarrow \mathbb{C}^{s_{k}}$ be the standard isometries as in the proof of Theorem 1. Define $\tilde{\alpha}_{k}, \tilde{\beta}_{k}$ $\in C^{s_{k}}$ by $\tilde{\alpha}_{k}=i_{s_{k}}\left(x_{k}\right), \tilde{\beta}_{k}=i_{s_{k}}\left(y_{k}\right)$. Consider the $s_{k} \times s_{k}$ matrix $\mathbb{A}_{s_{k}}$, where the $(p, r)$-element of the $s_{k} \times s_{k}$ matrix $\mathbb{A}_{s_{k}}$ is equal to $\left\langle A \phi_{p}, \phi_{r}\right\rangle$, for $p, r=1,2, \cdots, s_{k}$. A simple calculation shows that $\left\langle\mathbb{A}_{s_{k}} \tilde{\alpha}_{k}, \tilde{\beta}_{k}\right\rangle=\left\langle A x_{k}, y_{k}\right\rangle,\left\langle\tilde{\alpha}_{s_{k}}, \tilde{\beta}_{s_{k}}\right\rangle=\left\langle x_{k}, y_{k}\right\rangle$. Since $\left\langle A x_{k}\right.$, $\left.y_{k}\right\rangle \longrightarrow\langle A x, y\rangle$ as $k \longrightarrow \infty$ and $\left\langle x_{k}, y_{k}\right\rangle \longrightarrow\langle x, y\rangle=q$ as $k$ $\longrightarrow \infty$, we have $\left\langle\mathbb{A}_{s_{k}} \tilde{\alpha}_{k}, \tilde{\beta}_{k}\right\rangle \longrightarrow\langle A x, y\rangle$ as $k \longrightarrow \infty$. Hence, there exist $\lambda_{k} \in W_{q}\left(\mathbb{A}_{s_{k}}\right)$ such that $\lambda_{k} \longrightarrow \lambda$. In view of Lemma 2, this immediately gives $\lambda \in \bigcup_{j=1}^{\infty} \bar{W}_{q}\left(\mathbb{A}_{j}\right)$.

Remark 4. The hypotheses $P_{k}$ converge strongly to the identity operator I are (in general) necessary. It is easy to construct an example where $\lambda \in \bigcup_{j=1}^{\infty} \bar{W}_{q}\left(\mathbb{A}_{j}\right)$ is a strict subset of $W_{q}(A)$.

Example 1. Let $A$ be an operator matrix in $H=\ell^{2}(\mathbb{N})$, where $A=\operatorname{diag}\{1 / n\}_{n=1}^{\infty}\left\{\mathscr{L}_{k}: k \in \mathbb{N}\right\}$ be a nested family of subspace in $\ell^{2}(\mathbb{N})$, with $\mathscr{L}_{k}=\operatorname{span}\left\{e_{2}, \cdots, e_{k+1}\right\}$, where $e_{j}=j^{\text {th }}$ standard basis vector, and $r_{k}=\operatorname{span}\left\{\psi_{1}, \cdots, \psi_{k}\right\}$ where $\left(\psi_{k}\right)_{k=1}^{\infty}$ is any orthonormal sequence. Then, performing an analysis analogous to Theorem3, we see that $\left(A x_{k}, y_{k}\right)$ is not convergent to $(A x, y)$, unless $x$ is orthogonal to $e_{1}$.

Remark 5. We assume readers are familiar with basic notions and results about linear unbounded operators, as well as matrices of nonnecessarily bounded operators. Useful references are [7-9]. We call a few definitions though: a linear operator A with a domain $\mathscr{D}(A)$ contained in a Hilbert space $\mathscr{H}$ is said to be densely defined if $\mathscr{D}(A)=\mathscr{H}$. Say that a linear operator $A$ is closed if its graph $\Gamma_{A}$ is closed in $\mathscr{H} \oplus \mathscr{H}$. A linear operator $A$ is called closable if the closure $\bar{\Gamma}_{A}$ of its graph is the graph of some operators. A subspace $\mathscr{D} \subset \mathscr{D}(A)$ is called a core of a closable operator $A$ if $\left.A\right|_{D}$ is closable with closure $A$. The definition of the $q$-numerical range for bounded linear operators in Equation((2)) generalizes as follows to unbounded operator matrices $A$ with dense domain $\mathscr{D}(A)$.

Definition 6. For a linear operator $A$ with domain $\mathscr{D}(A) \subset \mathscr{H}$, we define the $q$-numerical range of $A$ for $0 \leq q \leq 1$ by

$$
\begin{gathered}
W_{q}(A)=\{\langle A x, y\rangle, \text { for some } x, y \in \mathscr{D}(A),\|x\|=1=\|y\|, \\
\langle x, y\rangle=q\} .
\end{gathered}
$$

Theorem 7. Let $A$ be an unbounded operator in $\mathscr{H}$. Let \{ $\left.\mathscr{L}_{k}: k \in \mathbb{N}\right\}$ be a nested family of spaces in $\mathscr{D}(A)$ given by $\mathscr{L}_{k}=\operatorname{span}\left\{\phi_{1}, \phi_{2}, \cdots, \phi_{k}\right\}$, where $\left\{\phi_{k}: k \in \mathbb{N}\right\}$ is orthonormal, and $\mathbb{A}_{k}$ be as in Equation((4)). Then, $W_{q}\left(\mathbb{A}_{k}\right) \subseteq W_{q}$ $(A)$, for $q \in(0,1]$.

Proof. Define an isometry $\pi: \mathscr{L}_{k} \rightarrow \mathbb{C}^{k}$ by $\pi\left(\zeta_{1} \phi_{1}+\zeta_{2} \phi_{2}+\right.$ $\left.\cdots+\zeta_{k} \phi_{k}\right):=\left(\zeta_{1}, \zeta_{2}, \cdots, \zeta_{k}\right)$, and $\pi\left(\gamma_{1} \phi_{1}+\gamma_{2} \phi_{2}+\cdots+\gamma_{k} \phi_{k}\right):=($ $\left.\gamma_{1}, \gamma_{2}, \cdots, \gamma_{k}\right)$. Suppose that $\lambda \in W_{q}\left(\mathbb{A}_{k}\right)$. Then, for some $\zeta, \gamma$ $\in \mathbb{C}^{k}$, with $\|\zeta\|=1=\|\gamma\|,(\zeta, \gamma)=q$ such that $\lambda=\left\langle\mathbb{A}_{k} \zeta, \gamma\right\rangle$. Choose $\xi, \eta \in \mathscr{L}_{k}$, such that $\pi(\xi)=\zeta, \pi(\eta)=\gamma$, and $\|\xi\|=1=$ 
$\|\eta\|$. Then, a direct computation shows that $\lambda=\langle A \xi, \eta\rangle$, where $\xi=\sum_{j=1}^{k} \zeta_{j} \phi_{j}$, and $\eta=\sum_{j=1}^{k} \gamma_{j} \phi_{j}$. Thus, $\lambda \in W_{q}(A)$.

The following lemma can be the proof in a similar fashion as Lemma 2.

Lemma 8. Let $\left\{\mathscr{L}_{k}: k \in \mathbb{N}\right\}$ and $\mathbb{A}_{k}$ be as in Theorem7. Given $q \in(0,1]$, then $W_{q}\left(\mathbb{A}_{k}\right) \subseteq W_{q}\left(\mathbb{A}_{\tau}\right)$, for $r \geq k$.

In the following result, we describe that the closure of the range $W_{q}(A)$ is approximated by $W_{q}\left(\mathbb{A}_{k}\right)$ under the assumption that the linear span of $\left\{\phi_{1}, \phi_{2}, \cdots\right\}$ is a core of $A$.

Theorem 9. Let $A$ be an unbounded operator in $\mathscr{H}$. Let \{ $\left.\mathscr{L}_{k}: k \in \mathbb{N}\right\}$ be a nested family of spaces in $\mathscr{D}(A)$ given by $\mathscr{L}_{k}=\operatorname{span}\left\{\phi_{1}, \phi_{2}, \cdots, \phi_{k}\right\}$, where $\left\{\phi_{k}: k \in \mathbb{N}\right\}$ is orthonormal, and $\mathbb{A}_{k}$ be as in Equation((4)). Then, $W_{q}^{-}(A)=$ $\bigcup_{k=1}^{\infty} \bar{W}_{q}\left(\mathbb{A}_{k}\right)$, for $q \in[0,1]$.

Proof. Since $\mathscr{C}=\operatorname{span}\left\{\phi_{1}, \phi_{2}, \cdots\right\}$ is a core of $A$, there exists a sequence $\left(x_{k}\right)_{k=1}^{\infty}$, with each $x_{k} \in \operatorname{span}\left\{\phi_{1}, \phi_{2}, \cdots, \phi_{s_{k}}\right\}$ for some $s_{k}>0$ such that $\left\|x-x_{k}\right\| \longrightarrow 0$ and $\left\|A x-A x_{k}\right\| \longrightarrow 0$. In a similar way, we may also find a sequence $\left(y_{k}\right)_{k=1}^{\infty}$, with each $y_{k} \in \operatorname{span}\left\{\phi_{1}, \phi_{2}, \cdots, \phi_{s_{k}}\right\}$ for some $s_{k}>0$, such that $\left\|y-y_{k}\right\|$ $\longrightarrow 0$ and $\left\|A y-A y_{k}\right\| \longrightarrow 0$, so this means that $\|\left\langle A x_{k}, y_{k}\right\rangle-\langle$ $A x, y\rangle \| 0$ as $k \longrightarrow \infty$ and $\left\|\left\langle x_{k}, y_{k}\right\rangle-q\right\| \longrightarrow 0$ as $k \longrightarrow \infty$. Fix $k>0$. Let $\pi: \operatorname{span}\left\{\phi_{1}, \phi_{2}, \cdots, \phi_{s_{k}}\right\} \longrightarrow \mathbb{C}^{s_{k}}$ be the standard isometries as in the proof of Theorem 7. Define $\tilde{\alpha}_{k}, \tilde{\beta}_{k}$ $\in C^{s_{k}}$ by $\tilde{\alpha}_{k}=\pi_{s_{k}}\left(x_{k}\right), \tilde{\beta}_{k}=\pi_{s_{k}}\left(y_{k}\right)$. Consider the $s_{k} \times s_{k}$ matrix $\mathbb{A}_{s_{k}}$, where the $(p, r)$-element of the $s_{k} \times s_{k}$ matrix $\mathbb{A}_{s_{k}}$ is equal to $\left\langle A \phi_{p}, \phi_{r}\right\rangle$, for $p, r=1,2, \cdots, s_{k}$. A simple calculation shows that $\left\langle\mathbb{A}_{s_{k}} \tilde{\alpha}_{k}, \tilde{\beta}_{k}\right\rangle=\left\langle A x_{k}, y_{k}\right\rangle$ and $\left\langle\tilde{\alpha}_{k}, \tilde{\beta}_{k}\right\rangle=\left\langle x_{k}, y_{k}\right\rangle$. Since $\|\langle A$ $\left.x_{k}, y_{k}\right\rangle-\langle A x, y\rangle \| \longrightarrow 0$ as $k \longrightarrow \infty$ and $\left\|\left\langle x_{k}, y_{k}\right\rangle-q\right\| \longrightarrow 0$ as $k \longrightarrow \infty$, this implies that $\left\|\left\langle\mathbb{A}_{s_{k}} \tilde{\alpha}_{k}, \tilde{\beta}_{k}\right\rangle-\langle A x, y\rangle\right\| \longrightarrow 0$ as $k$ $\longrightarrow \infty$. Hence, there exists $\lambda_{k} \in W_{q}\left(\mathbb{A}_{s_{k}}\right)$ such that $\lambda_{k} \longrightarrow$ $\lambda$. In view of Lemma 8 , this immediately gives $\lambda \epsilon$ $\bigcup_{k=1}^{\infty} \bar{W}_{q}\left(\mathbb{A}_{k}\right)$.

\section{Numerical Experiments on Differential Operator}

In this section, we study some concrete examples and demonstrate that, in spite of the results obtained in the previous section, practical computation of the $q$-numerical range of differential operator is very far from being straightforward. We define the inner product $\langle u, v\rangle$ to be linear in the first parameter and conjugate linear in the second parameter, and we consider the space of square-integrable functions, $L^{2}(\Omega, d x)$, where $\Omega$ is an interval in $\mathbb{R}$, a Hilbert space with inner product

$$
\langle u, v\rangle=\int_{\Omega} u \bar{v} d x
$$

The computations were performed in Matlab.
3.1. Application to Schrödinger Operator. In the Hilbert space $H:=L^{2}(0,1)$, we introduce the Schrödinger operator

$$
A=-\frac{d^{2}}{d x^{2}}+p
$$

(with bounded potential $p$ ) and the domain of $L$ is given by

$$
\mathscr{D}(A)=\left\{u \in H^{2}(0,1): u(0)=0=u(1)\right\} .
$$

\section{Remark 10.}

(i) Because $A$ is self-adjoint and bounded below with purely discrete spectrum, the eigenvalues of $A$ are given by

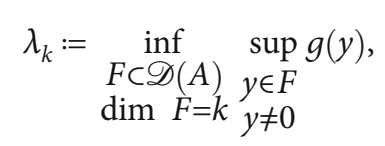

where $g$ is the Rayleigh functional

$$
g(y):=\frac{\langle A y, y\rangle}{\langle y, y\rangle}, \quad y \in \mathscr{D}(A), y \neq 0
$$

(ii) It is clear the operator $A$ in $L^{2}(0,1)$ has a sequence of eigenvalues and normalized eigenfunctions for the operator $A$ in $L^{2}(0,1)$ are

$$
\lambda_{n}=n^{2} \pi^{2}
$$

$$
\phi_{n}(x)=\sqrt{2} \sin (n \pi x) \quad \text { for } n=1,2,3, \cdots
$$

under the setting $p(x)=0$

(iii) Because Equation (7) is a closed operator, then it is not difficult to see that the subspace $\mathscr{C}_{A}=$ $\operatorname{span}\left\{\phi_{1}, \phi_{2}, \cdots\right\} \subset \mathscr{D}(A)$ is a core of $A$, so in this case, the main Theorem 9 is applicable to this example

(iv) We may use these eigenfunctions in Equation (12) as basis elements for a discretization of the type discussed in Section 2, form the matrix elements $\left\langle-\phi_{k}{ }^{\prime \prime}, \phi_{j}\right\rangle$, using the inner product in Equation (6) with respect to the orthonormal basis in Equation (12) and consider the (infinite) operator matrix 


$$
Q
$$

The matrix $\mathbb{A}_{k}$ in Equation (4) is obtained by taking the leading submatrices of the (infinite) operator matrix in Equation (13), with appropriate dimensions. Observe that $\left\langle-\phi_{k}{ }^{\prime}\right.$ $\left.{ }^{\prime}, \phi_{j}\right\rangle=\operatorname{diag}\left\{\pi^{2}, 4 \pi^{2}, 9 \pi^{2}, \cdots\right\}$ which can be evaluated explicitly. The following figure shows attempts to calculate $W_{q}\left(\mathbb{A}_{k}\right)$ for various $k$ and $q$ and also some attempts to estimate these sets by qualitative means, using existing theorems from the literature as well as the theorems proved above.

3.2. Analytical Estimates for Schrödinger Operator. In order to understand to what extent Figure 1 is qualitatively correct, we now analyze the $q$-numerical range of the Schrödinger operator. Comments:

(i) In Remark 10 part (i), it is obvious $\langle A y, y\rangle \geq \pi^{2}\langle y, y\rangle$; thus, the numerical range of $A$ is $W \overline{(A)}=\left[\pi^{2}, \infty\right)$

(ii) While for $0<q<1$, the $q$-numerical range $W_{q}(A)$ of $A$ contains the range

$$
W_{q}\left(\operatorname{diag}\left(n^{2}, n^{2} \pi^{2}\right)\right)
$$

for any integer $n \geq 2$. Let $\lambda=n^{2} \pi^{2}>0$. Then, the range

$$
\begin{aligned}
W_{q}\left(\operatorname{diag}\left(\pi^{2}, \lambda\right)\right)= & \left\{z=x+i y:(x, y) \in \mathbb{R}^{2},\left(\frac{x-q\left(\pi^{2}+\lambda\right)}{2}\right)^{2}\right. \\
& \left.+\frac{y^{2}}{1-q^{2}} \leq\left(\frac{\lambda-\pi^{2}}{2}\right)^{2}\right\}
\end{aligned}
$$

contains the convex polygon with vertexes

$$
\begin{gathered}
-(1-q) \frac{\lambda}{2},(1+q) \frac{\lambda}{2}, \\
-i\left(1-q^{2}\right) \frac{\lambda}{2}, i\left(1-q^{2}\right) \frac{\lambda}{2} .
\end{gathered}
$$

For a sufficiently large $\lambda$, such a convex polygon contains an arbitrary given point in the Gaussian plane. Thus, this differential operator satisfies $W_{q}(A)=\mathbb{C}$

(iii) If we restrict our attention for the variational approximation to the Schrödinger operator, we may have another story. The approximation $\mathbb{A}_{k}$ in Theorem 9 is given by a diagonal matrix with some real eigenvalues $\left.\left\{\lambda_{1}, \lambda_{2}, \cdots, \lambda_{k}\right)\right\}$ with $\lambda_{1} \leq \lambda_{2} \leq \cdots \leq$ $\lambda_{k}$. The $q$-numerical range $W_{q}\left(\mathbb{A}_{k}\right)$ of $\mathbb{A}_{k}$ for $0<q$ $<1$ is given by $W_{q}\left(\mathbb{A}_{k}\right)=\operatorname{diag}\left(\lambda_{1}, \lambda_{k}\right)$. Then, the range $W_{q}\left(\operatorname{diag}\left(\lambda_{1}, \lambda_{k}\right)\right)$ is the union of closed circular discs

$$
\begin{aligned}
W_{q}\left(\operatorname{diag}\left(\lambda_{1}, \lambda_{k}\right)\right)= & \left\{z=a+i b:(a, b) \in \mathbb{R}^{2},\left(a-\frac{q\left(\lambda_{1}+\lambda_{k}\right)}{2}\right)^{2}\right. \\
& \left.+\frac{b^{2}}{1-q^{2}} \leq\left(\frac{\left(\lambda_{k}-\lambda_{1}\right)}{2}\right)^{2}\right\}
\end{aligned}
$$

3.3. Application to Block Differential Operators. In this subsection, we apply Theorem 9 to compute the $q$-numerical range of Stokes-type operators and Hain-Lüst-type operators. First, we study Stokes-type operators.

3.3.1. Application to Stokes-Type Operators. Consider the differential expression in the Hilbert space $L_{2}^{2}(0,1):=L^{2}(0,1)$ $\oplus L^{2}(0,1)$, we introduce the matrix differential operator

$$
\mathscr{A}_{0}:=\left(\begin{array}{cc}
A_{0} & B_{0} \\
C_{0} & D_{0}
\end{array}\right)=\left(\begin{array}{cc}
-\frac{d^{2}}{d x^{2}} & -\frac{d}{d x} \\
\frac{d}{d x} & -\frac{3}{2}
\end{array}\right),
$$

on the domain

$$
\mathscr{D}\left(\mathscr{A}_{0}\right):=\left(H^{2}(0,1) \cap H^{1}(0,1)\right) \oplus H^{1}(0,1) .
$$

The operator matrix $\mathscr{A}_{0}$ is not closed but closable [2]. In order to show that its closure is self-adjoint, we need the following:

\section{Remark 11.}

(i) Consider the operator $M$ given by

$$
\begin{aligned}
\mathscr{D}(M):= & \left(\begin{array}{c}
y_{1} \\
y_{2}
\end{array}\right): y_{1} \in H_{0}^{1}(0,1), y_{1}^{\prime}+y_{2} \in H^{1}(0,1), \\
\text { and } \left.y_{2} \in L_{2}(0,1)\right\}, &
\end{aligned}
$$

such that

$$
M\left(\begin{array}{l}
y_{1} \\
y_{2}
\end{array}\right)=\left(\begin{array}{c}
-\left(y_{1}^{\prime}+y_{2}\right)^{\prime} \\
y_{1}^{\prime}-\frac{1}{2} y_{2}
\end{array}\right) .
$$

Lemma 12. The operator $M$ in Equation ((21)) coincides with the adjoint of the block operator matrix in Equation ((18)).

Proof. Suppose that

$$
g=\left(\begin{array}{l}
g_{1} \\
g_{2}
\end{array}\right) \in \mathscr{D}(\tilde{\mathscr{A}})
$$



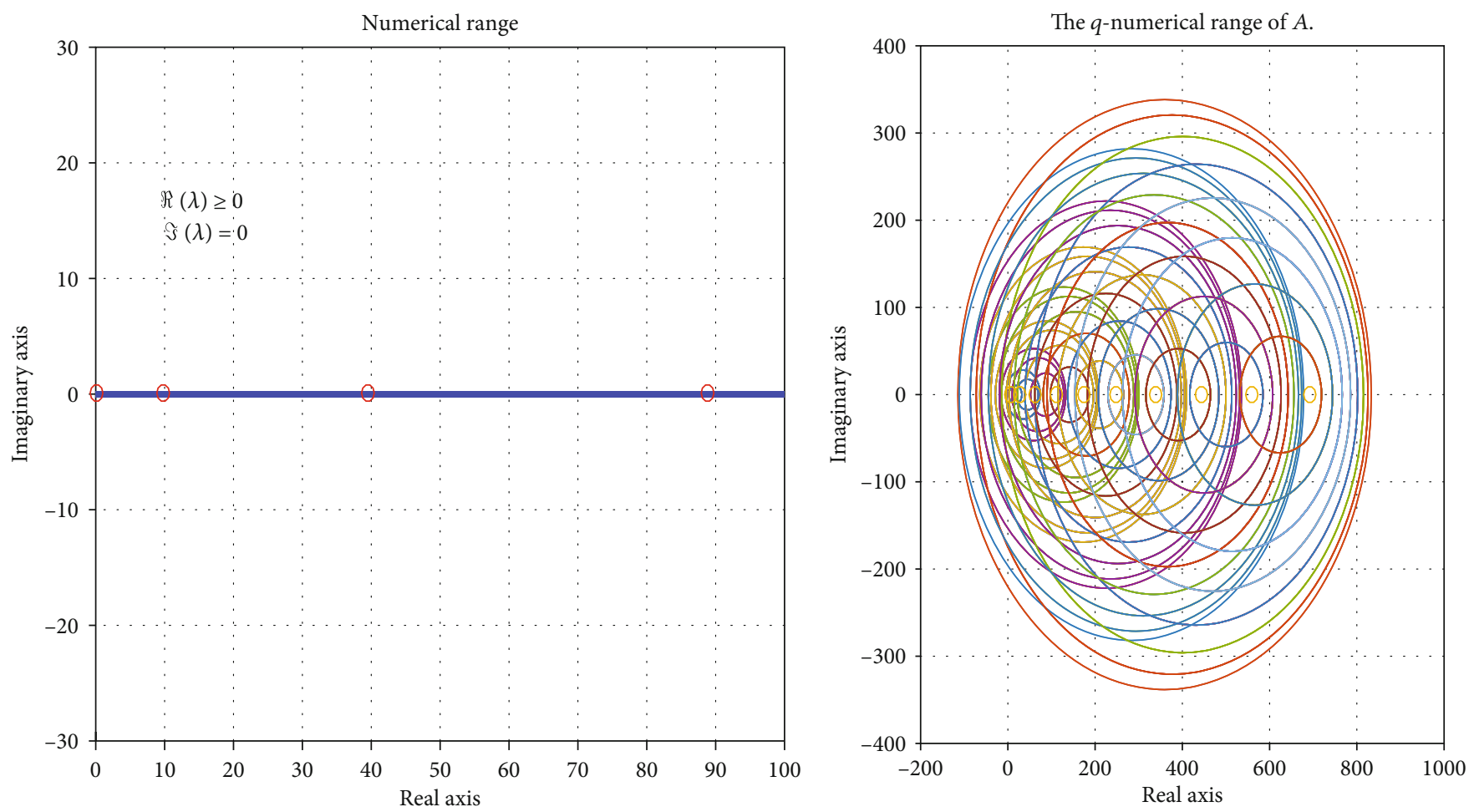

O Actual eigenvalues

FIgURE 1: On the left-hand side, the variational method approximation to the Schrödinger operator. The approximation $\mathbb{A}_{k}$ is given by a selfadjoint matrix with some real eigenvalues $\left\{\lambda_{1}, \lambda_{2}, \cdots, \lambda_{k}\right\}$ with $\lambda_{1} \leq \lambda_{2} \leq \cdots \lambda_{k}$. The $q$-numerical range $W_{q}\left(\mathbb{A}_{24}\right)$ of $\mathbb{A}_{24}$ for $q=1$ is the line segment $\left[\lambda_{1}, \lambda_{24}\right]$; the red dots are $\sigma\left(\mathbb{A}_{24}\right)$. On the right-hand side, the $q$-numerical range $W_{q}\left(\mathbb{A}_{24}\right)$ of $\mathbb{A}_{24}$ for $0<q<1$ is the union of the closed circular discs. The small red circles on the real axis inside the circular disk are $\sigma\left(\mathbb{A}_{24}\right)$ scaled by $q$.

with

$$
u=\left(\begin{array}{c}
u_{1} \\
u_{2}
\end{array}\right) \in L^{2}(0,1)^{2}
$$

Then

$$
\begin{aligned}
\left(\mathscr{A}_{0} y, g\right) & =(y, u), \quad y=\left(\begin{array}{l}
y_{1} \\
y_{2}
\end{array}\right) \in \mathscr{D}\left(\mathscr{A}_{0}\right) \\
& =\left(H^{2}(0,1) \cap H^{1}(0,1)\right) \oplus H^{1}(0,1)
\end{aligned}
$$

or, equivalently,

$$
\begin{aligned}
& \left(-\left(y_{1}^{\prime}+y_{2}\right)^{\prime}, g_{1}\right)_{L^{2}(0,1)}+\left(y_{1}^{\prime}-\frac{1}{2} y_{2}, g_{2}\right)_{L^{2}(0,1)} \\
& =\left(y_{1}, u_{1}\right)_{L^{2}(0,1)}+\left(y_{2}, u_{2}\right)_{L^{2}(0,1)}
\end{aligned}
$$

for all $y_{1}, y_{2} \in C_{0}^{\infty}(0,1)$ with compact support contained in $(0,1)$. In particular, when $y_{1}=0$, then Equation (25) becomes

$$
\left(-y_{2}^{\prime}, g_{1}\right)_{L^{2}(0,1)}+\left(-\frac{1}{2} y_{2}, g_{2}\right)_{L^{2}(0,1)}=\left(y_{2}, u_{2}\right)_{L^{2}(0,1)}
$$

The first part of the left-hand side of Equation (26) is a bounded linear functional of $y_{2}$, which means that $g_{1} \in H^{1}$ ( $0,1)$, and also, the second part of the left-hand side of equation (26) is a bounded linear functional of $y_{2}$, which means that $g_{2} \in L_{2}(0,1)$; again return to Equation (26), and integrating by part, we obtain

$$
\left(-y_{2}, g_{1}^{\prime}-\frac{1}{2} g_{2}\right)=\left(y_{2}, u_{2}\right) y_{2} \in C_{0}^{\infty}(0,1)
$$

Because $C_{0}^{\infty}(0,1)$ is a dense in $L_{2}(0,1)$, this means that $u_{2}=g_{1}^{\prime}-(1 / 2) g_{2}$. By the same argument, if we set $y_{2}=0$ $y_{1} \in H_{0}^{1}(0,1) \cap H^{2}(0,1)$, we find that

$$
\left(-y^{\prime \prime}{ }_{1}, g_{1}\right)_{L^{2}(0,1)}+\left(y_{1}^{\prime}, g_{2}\right)_{L^{2}(0,1)}=\left(y_{1}, u_{1}\right)_{L^{2}(0,1)} .
$$

Again, return to Equation (28), and integrating by part, we 
obtain

$$
\left[-y_{1}^{\prime}(x) g_{1} \overline{(x)}\right]_{x=0}^{x=1}+\left(-y_{1},\left(g_{1}^{\prime}+g_{2}\right)^{\prime}\right)=\left(y_{1}, u_{1}\right)
$$

The first part of the left-hand side of Equation (29) is bounded only when $g_{1}(0)=0=g_{1}(1)$, and the second part is bounded linear functional of $y_{1}$, which means that $g_{1}^{\prime}+$ $g_{2} \in H^{1}(0,1)$. Then, this implies that $g \in \mathscr{D}(\tilde{\mathscr{A}})$, and because $C_{0}^{\infty}(0,1)$ is a dense in $L_{2}(0,1)$, this means that $u_{1}=-$ $\left(g_{1}^{\prime}+g_{2}\right)^{\prime}$ on $(0,1)$. It follows that

$$
\left(\begin{array}{l}
u_{1} \\
u_{2}
\end{array}\right)=\tilde{\mathscr{A}}\left(\begin{array}{l}
g_{1} \\
g_{2}
\end{array}\right)
$$

\section{Remark 13.}

(i) By the same argument of Lemma 12, it is not difficult to see that the operator $\mathscr{A}_{0}$ in Equation (18) is symmetric in $L_{2}(0,1)^{2}$ with the domain

$$
\begin{gathered}
\mathscr{D}(M):=\left\{\left(\begin{array}{l}
y_{1} \\
y_{2}
\end{array}\right): y_{1} \in H_{0}^{1}(0,1), y_{1}^{\prime}+y_{2} \in H^{1}(0,1), \text { and } y_{2} \in L_{2}(0,1)\right\}, \\
M\left(\begin{array}{c}
y_{1} \\
y_{2}
\end{array}\right)=\left(\begin{array}{c}
-\left(y^{\prime}+y_{2}\right)^{\prime} \\
y_{1}^{\prime}-\frac{1}{2} y_{2}
\end{array}\right)
\end{gathered}
$$

(ii) Because $M$ is a symmetric operator and has nonempty resolvent, then $M$ self-adjoint. Thus, by [10, Theorem 5.4], $M=\overline{\mathscr{A}}_{0}$

The following result shows that the eigenvalues of the Stocks operator coincide with eigenvalues of the operator $M$.

Proposition 14. Let $\mathscr{A}_{0}$ be as in Equation((18)), then $\sigma_{p}\left(\overline{\mathscr{A}}_{0}\right.$ )$=\sigma_{p}\left(\mathscr{A}_{0}\right)$.

Proof. Let $\lambda \in \sigma_{p}\left(\overline{\mathscr{A}}_{0}\right)$, then there exists an $y=\left(y_{1} y_{2}\right)^{t} \in \mathscr{D}($ $\left.\overline{\mathscr{A}}_{0}\right), y \neq 0$, such that

$$
\overline{\mathscr{A}}_{0}\left(\begin{array}{l}
y_{1} \\
y_{2}
\end{array}\right)=\lambda\left(\begin{array}{l}
y_{1} \\
y_{2}
\end{array}\right)
$$

We see that Equation (32) is equivalent to the following system of equations:

$$
\begin{aligned}
& -\left(y_{1}^{\prime}+y_{2}\right)^{\prime}=\lambda y_{1}, \\
& y_{1}^{\prime}-\frac{1}{2} y_{2}=\lambda y_{2},
\end{aligned}
$$

where $\left(y_{1} y_{2}\right)^{t} \in \mathscr{D}\left(\overline{\mathscr{A}}_{0}\right)$ and

$$
\begin{aligned}
\mathscr{D}\left(\overline{\mathscr{A}}_{0}\right):= & \left\{\left(\begin{array}{l}
y_{1} \\
y_{2}
\end{array}\right): y_{1} \in H_{0}^{1}(0,1), y_{1}^{\prime}+y_{2} \in H^{1}(0,1),\right. \\
& \text { and } \left.y_{2} \in L_{2}(0,1)\right\} .
\end{aligned}
$$

Because $y_{1} \in H^{1}(0,1)$, then from Equation (33), we get $y_{1}^{\prime}+y_{2} \in H^{2}(0,1)$ and Equation (34) can be written as

$$
y_{1}^{\prime}+y_{2}=\left(\lambda+\frac{3}{2}\right) y_{2}
$$

this implies that $y_{2} \in H^{2}(0,1)$ for $\lambda \neq(-3 / 2)$. Since $H^{2}(0,1)$ is a linear space then $y_{1}^{\prime} \in H^{2}(0,1)$, hence $y_{1} \in H^{3}(0,1) \subseteq$ $H^{2}(0,1)$, it follows that $y=\left(y_{1}, y_{2}\right)^{t} \in \mathscr{D}\left(\mathscr{A}_{0}\right)$, so $\lambda$ is an eigenvalue of $\mathscr{A}_{0}$.

Conversely, if $\lambda \in \sigma_{p}(\mathscr{A})$, then there exists an $y=$ $\left(y_{1}, y_{2}\right)^{t} \in \mathscr{D}\left(\mathscr{A}_{0}\right) \subseteq \mathscr{D}\left(\overline{\mathscr{A}}_{0}\right), y \neq 0$, such that

$$
\mathscr{A}_{0}\left(\begin{array}{l}
y_{1} \\
y_{2}
\end{array}\right)=\lambda\left(\begin{array}{l}
y_{1} \\
y_{2}
\end{array}\right)
$$

This means that $y$ is an eigenfunction of $\overline{\mathscr{A}}_{0}$ since $\left.\overline{\mathscr{A}}_{0}\right|_{\mathscr{D}\left(\mathscr{A}_{0}\right)}=\mathscr{A}_{0}$.

Remark 15.

(i) It may be shown that $\mathscr{A}$ has two series of eigenvalues $\lambda_{k}^{ \pm}$given by

$$
\lambda_{k}^{ \pm}=\frac{k^{2} \pi^{2}-3 / 2 \pm \sqrt{\left(3 / 2-k^{2} \pi^{2}\right)^{2}+10 k^{2} \pi^{2}}}{2}, \quad k=1,2, \cdots
$$

The eigenvalues of the minus series are located in the interval $(-5 / 2,-3 / 2]$ and convergent to $-5 / 2$ as $k \longrightarrow \infty$. The eigenvalues of the plus series are located in the interval $[0, \infty)$ and convergent to $\infty$ as $k \longrightarrow \infty$. The essential spectrum of $\mathscr{A}$ is the accumulation point of the eigenvalues: $\sigma_{\text {ess }}(M)=\{-5 / 2\}$

(ii) It is not difficult to see that the subspace $\mathscr{C}_{0}:=\mathscr{C}_{A}$ $\oplus \mathscr{C}_{D} \subset \mathscr{D}\left(\mathscr{A}_{0}\right)=\left(\mathscr{D}\left(A_{0}\right) \cap \mathscr{D}\left(C_{0}\right)\right) \oplus\left(\mathscr{D}\left(B_{0}\right) \cap \mathscr{D}(\right.$ $\left.\left.D_{0}\right)\right)$ is a core of $\mathscr{A}_{0}$

(iii) Now form the matrix elements $\left\langle-\phi_{k}^{\prime}, \phi_{j}\right\rangle,\left\langle-\phi_{k}^{\prime}, \phi_{j}\right\rangle$, $\left\langle\phi_{k}^{\prime}, \phi_{j}\right\rangle,\left\langle-(3 / 2) \phi_{k}, \phi_{j}\right\rangle$ using the inner product in (6) with respect to the orthonormal basis in Equation (12) and consider the (infinite) block operator matrix 


$$
\mathscr{G}:=\left(\begin{array}{cc}
\left\langle-\phi_{k}^{\prime}, \phi_{j}\right\rangle & \left\langle-\phi_{k}^{\prime}, \phi_{j}\right\rangle \\
\left\langle\phi_{k}^{\prime}, \phi_{j}\right\rangle & \left\langle-\frac{3}{2} \phi_{k}, \phi_{j}\right\rangle
\end{array}\right) .
$$

The defined matrix $\mathbb{A}_{k}$ in (4) is obtained by taking the leading submatrices of the block $\mathscr{G}$, with appropriate dimensions. Observe that $\left\langle-\phi_{k}^{\prime}, \phi_{j}\right\rangle=\operatorname{diag}\left\{\pi^{2}, 4 \pi^{2}, 9 \pi^{2}\right.$, $\ldots\}$,

$$
\begin{aligned}
& \left\langle-\phi_{k}^{\prime}, \phi_{j}\right\rangle=\left(\begin{array}{ll}
0, & \text { ifk }=\mathrm{j} ; \\
-2 k \pi \int_{0}^{1} \cos (k \pi x) & \sin (j \pi x) d x, \quad \text { ifk } \neq \mathrm{j} ;
\end{array}\right.
\end{aligned}
$$

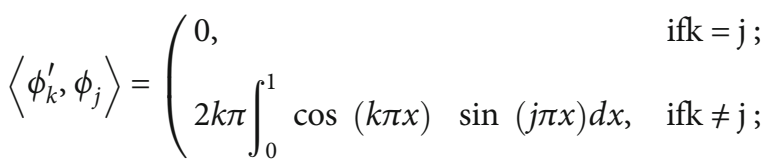

and $\left\langle-(3 / 2) \phi_{k}, \phi_{j}\right\rangle=-(3 / 2) \delta_{k, j}$, which can be evaluated explicitly

Figure 2 shows attempts to calculate $W_{q}\left(\mathbb{A}_{k}\right)$ for various $k$ and $q$ and also some attempts to estimate these sets by qualitative means, using existing theorems from the literature as well as the theorems proved above.

3.4. Analytical Estimates for Stokes Operator. In order to understand the results in Figure 2, it is useful to find an analytical estimate for $W_{q}(M)$. Let $\vec{\zeta}, \vec{\eta} \in \mathscr{D}(M)$, where

$$
\begin{aligned}
& \vec{\zeta}=\left(\begin{array}{l}
\zeta_{1} \\
\zeta_{2}
\end{array}\right), \\
& \vec{\eta}=\left(\begin{array}{l}
\eta_{1} \\
\eta_{2}
\end{array}\right)
\end{aligned}
$$

with $\|\vec{\zeta}\|=\|\vec{\eta}\|=1,\langle\vec{\zeta}, \vec{\eta}\rangle=q$ and let

$\lambda=\langle M \vec{\zeta}, \vec{\eta}\rangle=\left\langle-D^{2} \zeta_{1}, \eta_{1}\right\rangle+\left\langle-\zeta_{2}^{\prime}, \eta_{1}\right\rangle+\left\langle\zeta_{1}^{\prime}, \eta_{2}\right\rangle+\left\langle\alpha \zeta_{2}, \eta_{2}\right\rangle$,

where $\alpha=-(3 / 2)$. Equation (42) gives, as an estimate for the first term on the right-hand side of (42),

$$
\int_{0}^{1}-\zeta_{1}^{\prime} \bar{\eta}_{1} d x=\int_{0}^{1} \zeta_{1}^{\prime} \bar{\eta}_{1}^{\prime} d x \geq q \pi^{2} \int_{0}^{1} \zeta_{1} \bar{\eta}_{1} d x
$$

For the second and the third term on the right-hand side of (42), the Cauchy-Schwarz inequality and Youngs inequality yield

$$
\begin{gathered}
\operatorname{Re}\left(\int_{0}^{1}-\zeta_{2}^{\prime} \bar{\eta}_{1} d x\right)=\operatorname{Re}\left(\int_{0}^{1} \zeta_{2} \bar{\eta}_{1}{ }^{\prime} d x\right) \geq-\int_{0}^{1}\left(\left|\zeta_{2}\right|^{2}+\left|\eta_{1}{ }^{\prime}\right|^{2} d x\right), \\
\operatorname{Re}\left(\int_{0}^{1} \zeta_{1}^{\prime} \bar{\eta}_{2} d x\right) \geq-\int_{0}^{1}\left(\left|\zeta_{1}{ }^{\prime}\right|^{2}+\left|\eta_{2}\right|^{2} d x\right) .
\end{gathered}
$$

Combining Equations (44) and (45), we get that

$$
\operatorname{Re}\left(\int_{0}^{1} \zeta_{2}{ }^{\prime} \bar{\eta}_{1} d x\right)+\operatorname{Re}\left(\int_{0}^{1} \zeta_{1}{ }^{\prime} \bar{\eta}_{2} d x\right) \geq-q .
$$

The fourth term of the right-hand side of Equation (42) satisfies

$\operatorname{Re}\left(\int_{0}^{1} \alpha \zeta_{2} \bar{\eta}_{2} d x\right) \geq \inf \operatorname{Re}(\alpha)\left(q-\int_{0}^{1} \zeta_{1} \bar{\eta}_{1} d x\right)$.

Hence, from Equations (43), (46), and (47), we get that

$\operatorname{Re}(\lambda) \geq q \pi^{2} \int_{0}^{1} \zeta_{1} \bar{\eta}_{1} d x-q+\inf \operatorname{Re}(\alpha)\left(q-\int_{0}^{1} \zeta_{1} \bar{\eta}_{1} d x\right)$.

This simplifies to

$$
\begin{gathered}
\operatorname{Re}(\lambda) \geq q \pi^{2} \int_{0}^{1} \zeta_{1} \bar{\eta}_{1} d x-q+\left(q-\int_{0}^{1} \zeta_{1} \bar{\eta}_{1}\right) \inf \operatorname{Re}(\alpha) \\
=q\left(\pi^{2}-\inf \Re(\alpha)\right) \int_{0}^{1} \zeta_{1} \bar{\eta}_{1} d x+q(\inf \operatorname{Re}(\alpha)-1) .
\end{gathered}
$$

This yields

$$
\operatorname{Re}(\lambda) \geq \begin{cases}q(\alpha-1), & \text { if } \pi^{2}-\alpha \geq 0 ; \\ q\left(\pi^{2}-1\right), & \text { if } \pi^{2}-\alpha<0 .\end{cases}
$$

For our example, these yield $\operatorname{Re}(\lambda) \geq-(5 / 2) q$.

To estimate $\operatorname{Im}(\lambda)$, observe that

$$
\operatorname{Im}(\lambda)=\int_{0}^{1}(\operatorname{Im}(u)) \zeta_{2} \bar{\eta}_{2} d x=0 .
$$

This completes the estimates on $W_{q}(M)$.

3.5. The Hain-Lüst Operator. This operator was introduced by Hain and Lüst in application to problems of magnetohydrodynamics [11], and the problems of this type were studied in $[7,8,12]$. Assume that $w:[0,1] \rightarrow[0, \infty), \tilde{w}:[0,1] \rightarrow[0$, $\infty)$, and $u:[0,1] \rightarrow \mathbb{C}$ are such that $w(x)=1, \tilde{w}(x)=1, u($ $x)=18 e^{2 \pi i x}-20$, for each $x \in[0,1]$. We introduce the 

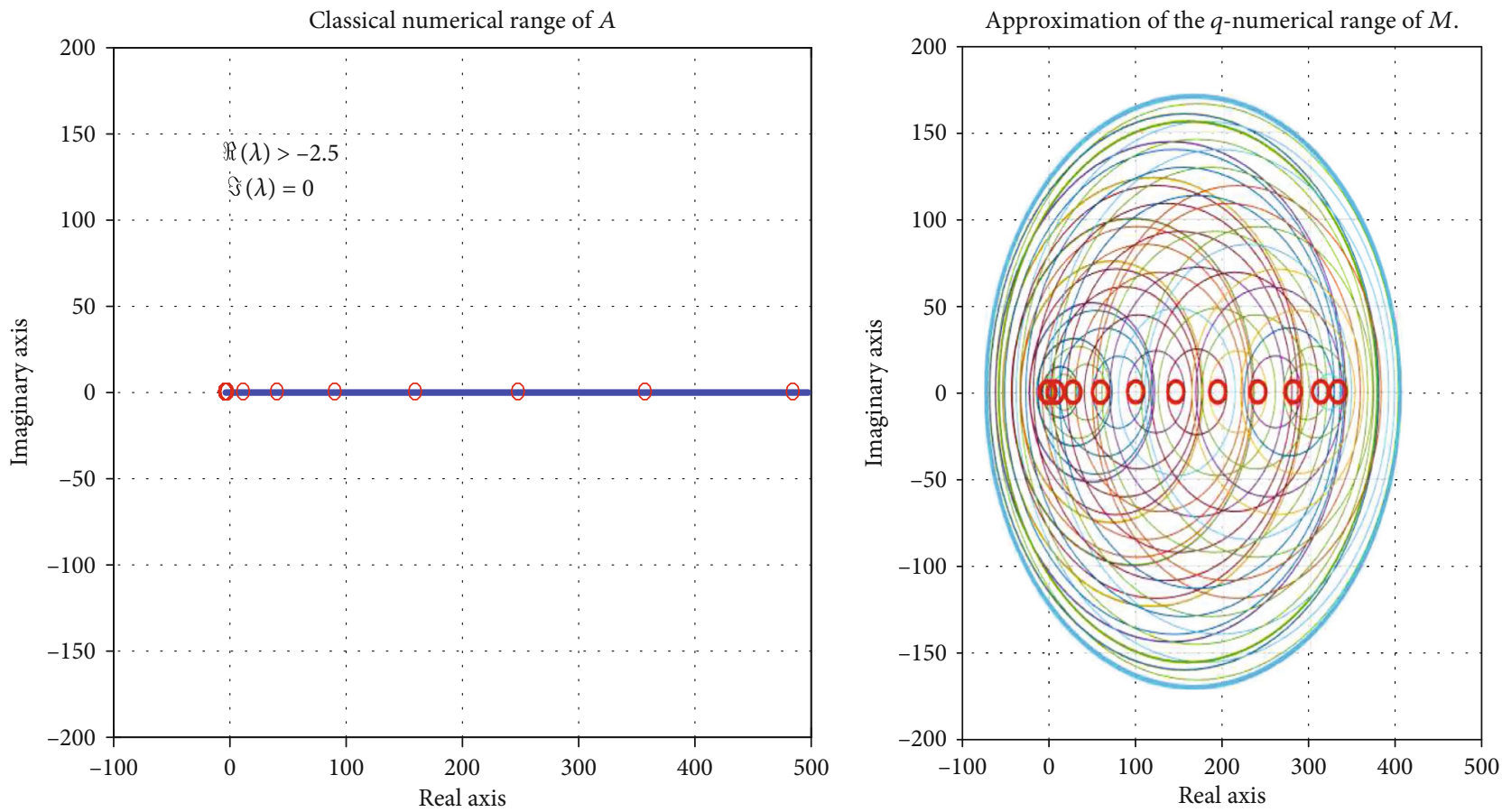

Figure 2: On the left-hand side, the variational method approximation to the Stokes operator. The approximation $\mathbb{A}_{k}$ is given by a real symmetric matrix with some eigenvalues $\left\{\lambda_{1}, \lambda_{2}, \cdots, \lambda_{28}\right\}$ with $\lambda_{1} \leq \lambda_{2} \leq \cdots \lambda_{28}$. The $q$-numerical range $W_{q}\left(\mathbb{A}_{28}\right)$ of $\mathbb{A}_{28}$ for $q=1$ is the line segment $\left[\lambda_{1}, \lambda_{28}\right]$; the red dots are $\sigma\left(\mathbb{A}_{28}\right)$. On the right-hand side, the q-numerical range $W_{q}\left(\mathbb{A}_{28}\right)$ of $\mathbb{A}_{28}$ for $0<q<1$ is the union of closed circular disk center at $q x^{*} \mathbb{A}_{28} x$ with radius $\sqrt{1-q^{2}} r(x)$ where $r(x)=\sqrt{\left\|\mathbb{A}_{28} x\right\|^{2}-\left|x * \mathbb{A}_{28} x\right|^{2}}$. The small red circles inside the circular disk are $\sigma\left(\mathbb{A}_{28}\right)$ scaled by $q$.

differential expression

$$
\begin{aligned}
\tau_{\tilde{A}} & :=-\frac{d^{2}}{d x^{2}}, \\
\tau_{\tilde{B}} & :=w(x), \\
\tau_{\tilde{C}} & :=\tilde{w}(x), \\
\tau_{\tilde{D}} & :=u(x) .
\end{aligned}
$$

Let $A, B, C, D$ be the operators in the Hilbert space $L^{2}(0$ ,1) induced by the differential expressions $\tau_{\tilde{A}}, \tau_{\tilde{B}}, \tau_{\tilde{C}}$, and $\tau_{\tilde{D}}$ with domain

$$
\begin{gathered}
\mathscr{D}(A):=H^{2}(0,1) \cap H_{0}^{1}(0,1), \\
\mathscr{D}(B)=\mathscr{D}(C)=\mathscr{D}(D):=L^{2}(0,1) .
\end{gathered}
$$

In the Hilbert space $L_{2}^{2}(0,1):=L^{2}(0,1) \oplus L^{2}(0,1)$, we introduce the matrix differential operator

$$
\mathscr{A}:=\left(\begin{array}{ll}
A & B \\
C & D
\end{array}\right)=\left(\begin{array}{cc}
-\frac{d^{2}}{d x^{2}} & w(x) \\
\tilde{w}(x) & u(x)
\end{array}\right),
$$

on the domain

$$
\mathscr{D}(\mathscr{A}):=\left(H^{2}(0,1) \cap H_{0}^{1}(0,1)\right) \oplus L^{2}(0,1) .
$$

\section{Remark 16.}

(i) By [13], Corollary VII.2.7, the operator $A:=-\left(d^{2} / d\right.$ $\left.x^{2}\right)$ with domain $\mathscr{D}(A):=\left(H^{2}(0,1) \cap H_{0}^{1}(0,1)\right)$ is closed. Moreover, because $\mathscr{D}(A) \subset \mathscr{D}(C)$, then the operator $C$ is $A$-bounded with relative bound 0 . This follows since there is a $\gamma>0$ such that, for every $\varepsilon>$ 0 ,

$$
\|C f\|^{2} \leq \gamma\|\langle A f, f\rangle\| \leq \gamma\left(\varepsilon\|A f\|^{2}+\varepsilon^{-1}\|f\|^{2}\right) .
$$

On the other hand $\mathscr{D}(D) \subset \mathscr{D}(B)$, then the operator $B$ is $D$ -bounded, we conclude that the operator matrix $D(A)$ is diagonally dominant of order 0 ; it is closed by [14], Corollary 2.2.9 (i)

(ii) In Equation (54), since $A$ is self-adjoint with the purely discrete spectrum, then the linear span $\mathscr{C}_{A}$ $=\operatorname{span}\left\{\phi_{1}, \phi_{2}, \cdots\right\}$ is a core of $A$, where $\left\{\phi_{k}: k \epsilon\right.$ $\mathbb{N}\}$ is an orthonormal basis in $L^{2}(0,1)$, and by the same argument because $D$ in Equation (54) is 
bounded, then the linear span $\mathscr{C}_{D}=\operatorname{span}\left\{\phi_{1}, \phi_{2}\right.$, $\ldots\}$ is a core of $D$. Hence, it is not difficult to see that the subspace $\mathscr{C}:=\mathscr{C}_{A} \oplus \mathscr{C}_{D} \subset \mathscr{D}(\mathscr{A})=(\mathscr{D}(A) \cap \mathscr{D}($ $C)) \oplus(\mathscr{D}(B) \cap \mathscr{D}(D))$ is a core of $\mathscr{A}$. So the main Theorem 9 is applicable to this example

(iii) We may use the eigenfunctions in Equation (12) as basis elements for a discretization of the type discussed in Section 2, forming the matrix elements $<$ $\left.A \phi_{k}, \phi_{j}\right\rangle,\left\langle w \phi_{k}, \phi_{j}\right\rangle,\left\langle\tilde{w} \phi_{k}, \phi_{j}\right\rangle,\left\langle u \phi_{k}, \phi_{j}\right\rangle$, with respect to the inner product in (6) and considering the infinite block matrix

$$
Q=\left(\begin{array}{cc}
\left\langle A \phi_{k}, \phi_{j}\right\rangle & \left\langle w \phi_{k}, \phi_{j}\right\rangle \\
\left\langle\tilde{w} \phi_{k}, \phi_{j}\right\rangle & \left\langle u \phi_{k}, \phi_{j}\right\rangle
\end{array}\right)
$$

The defined matrix $\mathbb{A}_{k}$ in (4) is obtained by taking the leading submatrices of the block $\mathbb{Q}$, with appropriate dimensions.

Observe that $\left\langle A \phi_{k}, \phi_{j}\right\rangle=\operatorname{diag}\left\{\pi^{2}, 4 \pi^{2}, 9 \pi^{2}, \cdots\right\}$, $\left\langle w \phi_{k}, \phi_{j}\right\rangle=\operatorname{diag}\{1,1,1, \cdots\},\left\langle\tilde{w} \phi_{k}, \phi_{j}\right\rangle=\operatorname{diag}\{1,1,1, \cdots\}$, and $\left\langle u \phi_{k}, \phi_{j}\right\rangle=36 \int_{0}^{1} e^{2 \pi i x} \sin (k \pi x) \sin (j \pi x) d x-20 \delta_{k, j}$, which can be evaluated explicitly. If the operator $A$ included a potential, for instance, then its eigenfunctions would not generally be explicitly computable. We could still use the functions $\phi_{j}$ in (12) as basis functions, but the matrix elements $\left\langle A \phi_{k}, \phi_{j}\right\rangle$ would have to be computed by quadrature and the corresponding matrix would no longer diagonal. Figure 3 shows attempts to compute the numerical approximation of the boundary of $W_{q}\left(\mathbb{A}_{k}\right)$ for various $k$ and $q$ and also some attempts to estimate these sets by qualitative means, using existing theorems from the literature as well as the theorems proved above.

3.6. Analytical Estimates for Non-Self-Adjoint Hain-Lüst Operator. In order to understand the results in Figure 3, it is useful to find an analytical estimate for $W_{q}(\mathscr{A})$. Let $\vec{x}, \vec{y}$ $\in \mathscr{D}(\mathscr{A})$, where

$$
\begin{gathered}
\vec{x}=\left(\begin{array}{l}
x_{1} \\
x_{2}
\end{array}\right), \\
\vec{y}=\left(\begin{array}{l}
y_{1} \\
y_{2}
\end{array}\right)
\end{gathered}
$$

with $\|\vec{x}\|=\|\vec{y}\|=1,(\vec{x}, \vec{y})=q$ and let

$\lambda=\langle\mathscr{A} \vec{x}, \vec{y}\rangle=\left\langle-D^{2} x_{1}, y_{1}\right\rangle+\left\langle x_{2}, y_{1}\right\rangle+\left\langle x_{1}, y_{2}\right\rangle+\left\langle u x_{2}, y_{2}\right\rangle$.
The first term of (59) gives, as an estimate,

$$
\int_{0}^{1} x_{1}{ }^{\prime \prime} \bar{y}_{1} d x \geq q \pi^{2} \int_{0}^{1} x_{1} \bar{y}_{1} d x
$$

For the second and the third term on the right-hand side of (59), the Cauchy-Schwarz inequality and Youngs inequality yield

$$
\begin{array}{r}
\mathfrak{R}\left(\int_{0}^{1} x_{2} \bar{y}_{1} d x\right) \geq-\int_{0}^{1}\left(\left|x_{2}\right|^{2}+\left|y_{1}\right|^{2}\right) d x, \\
\Re\left(\int_{0}^{1} x_{1} \bar{y}_{2} d x\right) \geq-\int_{0}^{1}\left(\left|x_{1}\right|^{2}+\left|y_{2}\right|^{2}\right) d x .
\end{array}
$$

Combining Equations (61) and (62), we get that

$$
\mathfrak{R}\left(\int_{0}^{1} x_{2} \bar{y}_{1} d x\right)+\mathfrak{R}\left(\int_{0}^{1} x_{1} \bar{y}_{2} d x\right) \geq-q
$$

The fourth term of the right-hand side of Equation (59) satisfies

$$
\mathfrak{R}\left(\int_{0}^{1} u x_{2} \bar{y}_{2} d x\right) \geq \inf \mathfrak{R}(u)\left(q-\int_{0}^{1} x_{1} \bar{y}_{1} d x\right) .
$$

Hence, from Equations (60), (63), and (64), we get that

$$
\mathfrak{R}(\lambda) \geq q \pi^{2} \int_{0}^{1} x_{1} \bar{y}_{1} d x-q+\inf \mathfrak{R}(u)\left(q-\int_{0}^{1} x_{1} \bar{y}_{1} d x\right) .
$$

This simplifies to

$$
\begin{aligned}
\mathfrak{R}(\lambda) & \geq q \pi^{2} \int_{0}^{1} x_{1} \bar{y}_{1}-q+\left(q-\int_{0}^{1} x_{1} \bar{y}_{1}\right) \text { inf } \mathfrak{R}(u) \\
& =q\left(\pi^{2}-\inf \mathfrak{R}(u)\right) \int_{0}^{1} x_{1} \bar{y}_{1} d x+q(\inf \mathfrak{R}(u)-1) .
\end{aligned}
$$

This yields

$\mathfrak{R}(\lambda) \geq \begin{cases}q(\inf \mathfrak{R}(u)-1), & \text { if } \pi^{2}-\inf \mathfrak{R}(u) \geq 0 ; \\ q\left(\pi^{2}-1\right), & \text { if } \pi^{2}-\inf \mathfrak{R}(u)<0 .\end{cases}$

For our example, these yield $\mathfrak{R}(\lambda) \geq-53 q$. 

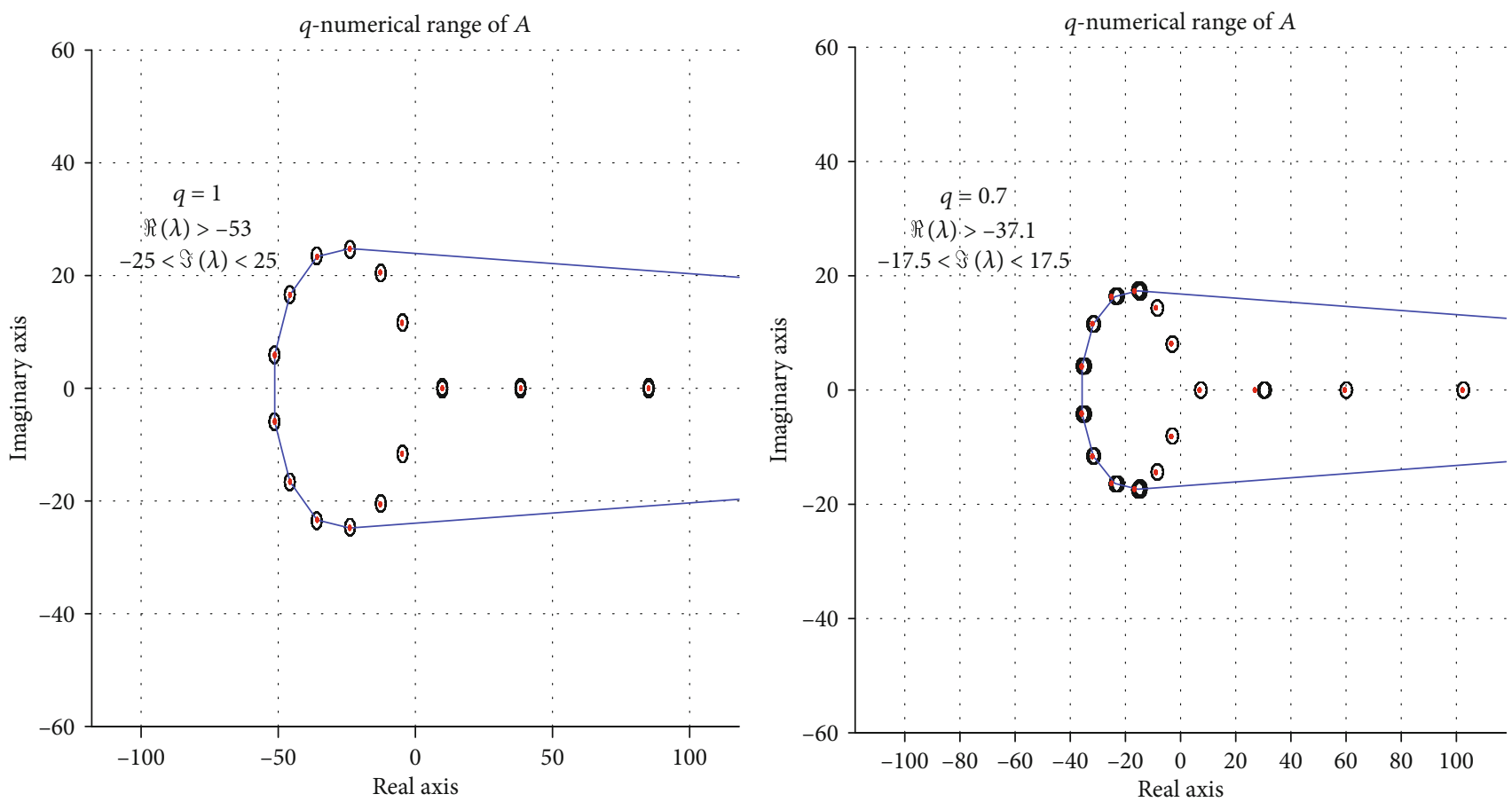

FIGURE 3: On the left-hand side, the variational method approximation to the Hain-Lüst operator for computing $q$-numerical range $W_{q}\left(\mathbb{A}_{48}\right)$ of $\mathbb{A}_{48}$ for $q=1$. The red dots are $\sigma\left(\mathbb{A}_{48}\right)$. On the right-hand side, for $0<q<1$, the variational method approximation for computing $W_{q}\left(\mathbb{A}_{48}\right)$ of $\mathbb{A}_{48}$ for $0<q<1$. The red dots are $\sigma\left(\mathbb{A}_{48}\right)$ scaled by $q$.

To estimate $\operatorname{Im}(\lambda)$, observe that

$\operatorname{Im}(\lambda)=\int_{0}^{1}(\operatorname{Im}(u)) x_{2} \bar{y}_{2} d x \leq \sup _{x \in[0,1]}(\operatorname{Im}(u)) \int_{0}^{1} x_{1} \bar{y}_{1} d x \leq q 25$,

$\operatorname{Im}(\lambda)=\int_{0}^{1}(\operatorname{Im}(u)) x_{2} \bar{y}_{2} d x \geq \inf _{x \in[0,1]}(\operatorname{Im}(z)) \int_{0}^{1} x_{2} \bar{y}_{2} d x \geq-q 25$,

hence

$$
-q 25 \leq \operatorname{Im}(\lambda) \leq q 25 .
$$

This completes the estimates on $W_{q}(A)$.

The following result shows that the $q$-numerical range of Example 1, is unbounded.

Remark 17. Suppose that

$$
\begin{aligned}
& \vec{x}=\left(\begin{array}{c}
\sqrt{2} \sin (k \pi x) \\
0
\end{array}\right), \\
& \vec{y}=\left(\begin{array}{c}
\sqrt{2} \sin (k \pi x) \\
0
\end{array}\right)
\end{aligned}
$$

where $\vec{x}, \vec{y} \in \mathscr{D}(A)$, with $\|\vec{x}\|=\|\vec{y}\|=1$, and $(\vec{x}, \vec{y})=q$ then

$$
\langle A \vec{x}, \vec{y}\rangle=(\sqrt{2})^{2}(k \pi)^{2} \int_{0}^{1} \sin ^{2}(k \pi x) d x=k^{2} \pi^{2}
$$

But $k \in \mathbb{Z}$ can be arbitrarily large. This means that $\mathfrak{R}(\lambda)$ is unbounded above.

3.6.1. Application to Self-Adjoint Hain-Lüst Operator. In the Hilbert space $H=L^{2}(-\pi, \pi) \oplus L^{2}(-\pi, \pi)$, we introduce the matrix differential operator

$$
\tilde{\mathcal{A}}:=\left(\begin{array}{ll}
L & B \\
C & V
\end{array}\right)=\left(\begin{array}{cc}
-\frac{d^{2}}{d x^{2}}+4 & 1 \\
1 & z
\end{array}\right),
$$

where

$$
z(x)= \begin{cases}0, & \text { for }-\pi \leq \mathrm{x}<0 \\ 2, & \text { for } 0 \leq \mathrm{x}<\pi\end{cases}
$$

on the domain

$$
\mathscr{D}(\tilde{\mathscr{A}}):=\left(H^{2}(-\pi, \pi) \cap H_{0}^{1}(-\pi, \pi)\right) \oplus L^{2}(-\pi, \pi) .
$$



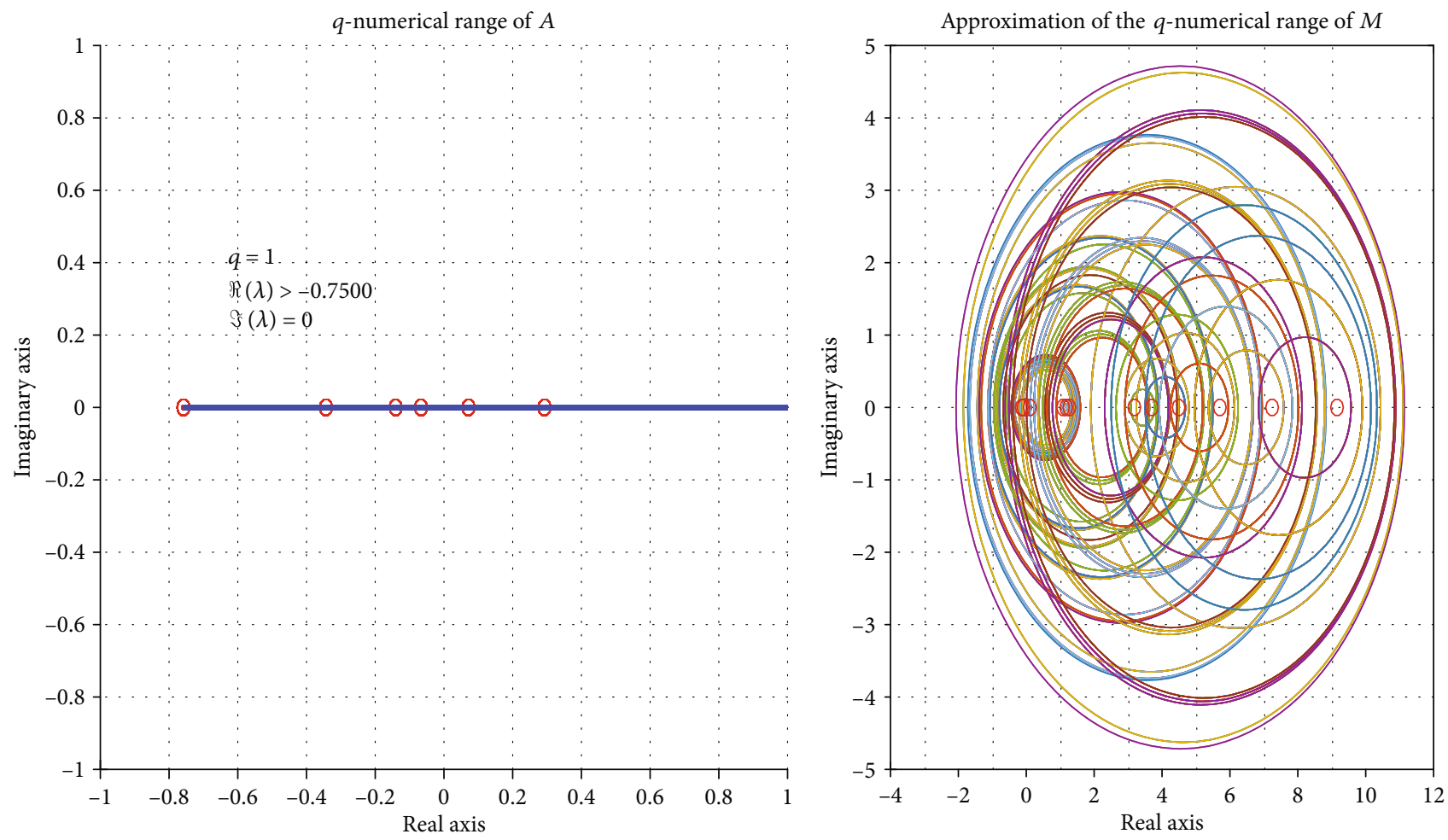

FIgURE 4: On the left-hand side, the variational method approximation to the Hain-Lüst operator. The approximation $\mathbb{A}_{k}$ is given by a real symmetric matrix with some eigenvalues $\left\{\lambda_{1}, \lambda_{2}, \cdots, \lambda_{28}\right\}$ with $\lambda_{1} \leq \lambda_{2} \leq \cdots \lambda_{28}$. The $q$-numerical range $W_{q}\left(\mathbb{A}_{28}\right)$ of $\mathbb{A}_{28}$ for $q=1$ is the line segment $\left[\lambda_{1}, \lambda_{28}\right]$; the red dots are $\sigma\left(\mathbb{A}_{28}\right)$. On the right-hand side, the $q$-numerical range $W_{q}\left(\mathbb{A}_{28}\right)$ of $\mathbb{A}_{28}$ for $0<q<1$ is the union of closed circular disk center at $q x^{*} \mathbb{A}_{28} x$ with radius $\sqrt{1-q^{2}} r(x)$ where $r(x)=\sqrt{\left\|\mathbb{A}_{28} x\right\|^{2}-\left|x * \mathbb{A}_{28} x\right|^{2}}$. The small red circles inside the circular disk are $\sigma\left(\mathbb{A}_{28}\right)$ scaled by $q$.

(i) It is not difficult to see that the subspace $\mathscr{C}:=\mathscr{C}_{A} \oplus$ $\mathscr{C}_{V} \subset \mathscr{D}(\tilde{\mathscr{A}})=(\mathscr{D}(L) \cap \mathscr{D}(C)) \oplus(\mathscr{D}(B) \cap \mathscr{D}(V))$ is a core of $\tilde{\mathscr{A}}$. So the main Theorem 9 is applicable to this example

(ii) The eigenvalues and normalized eigenfunctions of the operator $-d^{2} / d x^{2}$ in $L^{2}(-\pi, p i)$ are

$$
\begin{gathered}
\lambda_{j}=\frac{j^{2}}{4}, \\
\psi_{j}(x)=\frac{\sin (j(x+\pi) / 2)}{\sqrt{\pi}} \quad \text { for } j=1,2,3, \cdots
\end{gathered}
$$

Now form the matrix elements $\left\langle A \psi_{k}, \psi_{j}\right\rangle,\left\langle B \psi_{k}, \psi_{j}\right\rangle,\langle C$ $\left.\psi_{k}, \psi_{j}\right\rangle,\left\langle D \psi_{k}, \psi_{j}\right\rangle$, with respect to orthonormal basis $\psi_{j}(x)$ $=(1 / \sqrt{\pi}) \quad \sin (j(x+\pi) / 2)$ where $j \in \mathbb{N}$ and consider the infinite block operator matrix

$$
\mathscr{P}:=\left(\begin{array}{ll}
\left(A \psi_{k}, \psi_{j}\right) & \left(B \psi_{k}, \psi_{j}\right) \\
\left(C \psi_{k}, \psi_{j}\right) & \left(D \psi_{k}, \psi_{j}\right)
\end{array}\right) .
$$

The defined matrix $\mathbb{A}_{k}$ in (4) is obtained by taking the leading submatrices of the block $\mathscr{P}$, with appropriate dimensions. Observe that $\left\langle L \psi_{k}, \psi_{j}\right\rangle=\operatorname{diag}\{1 / 4,1 / 2, \cdots\}$, $\left\langle B \psi_{k}, \psi_{j}\right\rangle=\left\langle C \psi_{k}, \psi_{j}\right\rangle=\operatorname{diag}\{1,1,1, \cdots\}$, and

$$
\left\langle V \psi_{k}, \psi_{j}\right\rangle=\left(\begin{array}{ll}
\frac{1}{\pi} \int_{0}^{\pi} \sin \left(\frac{j(x+\pi)}{2}\right) \sin \left(\frac{k(x+\pi)}{2}\right), & \text { if } k \neq j ; \\
\frac{1}{2}, & \text { if } k=j ;
\end{array}\right.
$$

which can be evaluated explicitly. Figure 4 shows attempts to calculate $W_{q}\left(\mathbb{A}_{k}\right)$ for various $k$ and $q$ and also some attempts to estimate these sets by qualitative means, using existing theorems from the literature as well as the theorems proved above.

3.7. Analytical Estimates for Self-Adjoint Hain-Lüst Operator. In order to understand the results in Figure 4, it is useful to find an analytical estimate for $W_{q}(\tilde{\mathscr{A}})$. By the same arguments in Section 3.6, it is not difficult to see that $\operatorname{Re}(\lambda) \geq$ $-(3 / 4) q$, and

$$
\left.\operatorname{Im}(\lambda)=\int_{-\pi}^{\pi}(\operatorname{Im}(z)) x_{2} \overline{y_{2}} d x\right)=0
$$




\section{Conclusions}

This paper illustrates the practical difficulties associated with the computation of $q$-numerical ranges of operator matrices and block operator matrices of differential operators, even when good theoretical results are available to underpin the approximation procedure.

\section{Data Availability}

No data were used to support this study.

\section{Conflicts of Interest}

The authors declare that they have no conflicts of interest.

\section{References}

[1] F. Hausdorff, "Der Wertvorrat einer Bilinearform," Mathematische Zeitschrift, vol. 3, no. 1, pp. 314-316, 1919.

[2] A. Muhammad, Approximation of quadratic numerical range of block operator matrices, [Ph.D. thesis], Cardiff University, 2012.

[3] P. Andersen and M. Marcus, "Constrained extrema of bilinear functionals," Monatshefte für Mathematik, vol. 84, pp. 219235, 1977.

[4] N. K. Tsing, "The constrained bilinear form and C-numerical range," Linear Algebra and its Applications, vol. 56, pp. 195206, 1984.

[5] K. E. Gustafson and D. K. M. Rao, Numerical Range. The Field of Values of Linear Operators and Matrices, Universitext. Springer, New York, 1997.

[6] F. D. Muranghan, "On the field of values of a square matrix," Proceedings of the National Academy of Sciences, vol. 18, no. 3, pp. 246-248, 1932.

[7] H. Langer and C. Tretter, "Spectral decomposition of some nonselfadjoint block operator matrices," Journal of Operator Theory, vol. 39, pp. 339-359, 1998.

[8] H. Langer, R. Mennicken, and M. Möller, "A second order differential operator depending nonlinearly on the eigenvalue parameter," Operator theory Advances and Applications, vol. 48, pp. 319-332, 1990.

[9] N. Bebiano and J. da Providência, "Numerical ranges in physics," Linear and Multilinear Algebra, vol. 43, pp. 327-337, 1998.

[10] J. Weidmann and J. da Providência, Linear operators in Hilbert spaces, Springer-Verlag, New York-Berlin, 1980.

[11] K. Hain and R. Lüst, "Zur Stabilität zylindersymmetrischer plasmakonfigurationen mit volumenströmen," Zeitschrift für Naturforschung A, vol. 13, no. 11, pp. 936-940, 1958.

[12] V. M. Adamjan and H. Langer, "Spectral properties of a class of rational operator valued functions," Journal of Operator Theory, vol. 33, pp. 259-277, 1995.

[13] D. E. Edmunds and W. D. Evans, Spectral Theory and Differential Operators, Oxford University Press, New York, 1987.

[14] C. Tretter, Spectral Theory of Block Operator Matrices and Applications, Imperial College Press, London, 2008. 